\title{
Analytical Expressions for the Distortion of Asynchronous Sigma Delta Modulators
}

Amir Babaie-Fishani, Bjorn Van-Keymeulen and Pieter Rombouts

This document is an author's draft version submitted for publication to IEEE Trans. Circ. Syst.-II.

The actual version was published as [1].

\section{REFERENCES}

[1] A. Babaie-Fishani, B. Van Keymeulen, and P. Rombouts, "Analytical Expressions for the Distortion of Asynchronous Sigma-Delta Modulators," IEEE Trans. Circuits Syst.-II: Express Briefs, vol. 60, no. 8, pp. 472-476, 2013. 


\title{
Analytical Expressions for the Distortion of Asynchronous Sigma Delta Modulators
}

\author{
Amir Babaie-Fishani, Bjorn Van Keymeulen and Pieter Rombouts
}

\begin{abstract}
This work investigates the commonly used Asynchronous Sigma Delta Modulator which consists of a schmitttrigger and a continuous time loop filter. A detailed analysis is presented to accurately predict the distortion of such modulators. The extracted expressions are compared with simulation results and they illustrate an excellent match. The results are also compared with a previous work by Roza and they show a drastic improvement in accuracy.
\end{abstract}

Index Terms-Pulse Width Modulator, Asynchronous Sigma Delta Modulator, third order harmonic distortion.

\section{INTRODUCTION}

$\mathbf{P}$ ULSE Width Modulation techniques ${ }^{1}$ are growing popular in the design of Analog to Digital Converters (ADCs), [1]-[4], and switching Power Amplifiers (PAs) [5]. Such a representation is very convenient for low voltage environments and the corresponding two-level output signal can easily be processed by simple (pseudo-digital) circuits [3], [6].

Asynchronous Sigma Delta Modulators (ASDMs) are a known family of self-oscillating Pulse Width Modulators (PWMs), that can be implemented using a schmitt-trigger and a continuous time loop filter [7]-[9]. The purpose of this paper is to find analytical expressions that can predict the distortion of such modulators and their carrier frequency (the frequency at which they oscillate). Such expressions give insight to the design and understanding of the ASDMs and can be easily used for modelling and optimizing ASDMs regarding their linearity and switching activity.

There has been related prior work on the distortion of PWMs, e.g. [8]-[11]. However, [10] and [11] concern PWM structures that deviate from the class that is studied in this manuscript. The PWMs studied in [10] are ASDMs that have a continuous time loop filter but instead of a schmitt-trigger they have a comparator in the loop. In Such ASDMs, the order of the loop filter needs to be greater than two to have a stable oscillation. In the family of ASDMs that we are studying here, it is required that the hysteresis of the schmitt trigger is nonzero. The resulting ASDMs can already work with a passive 1 st order loop filter.

The authors are with the Department Electronics and information Systems, Ghent University, Ghent, Belgium.

This work has been supported by the Fund for Scientific Research Flanders (FWO-Vlaanderen) Belgium.

Copyright (c) 2013 IEEE. Personal use of this material is permitted. However, permission to use this material for any other purposes must be obtained from the IEEE by sending an email to pubs-permissions@ieee.org

${ }^{1}$ Here the term Pulse Width Modulation is used for any system that converts a baseband input signal into a two-level continuous time waveform of which the duty cycle represents the input signal. In particular, the modulation frequency is not necessarily constant.

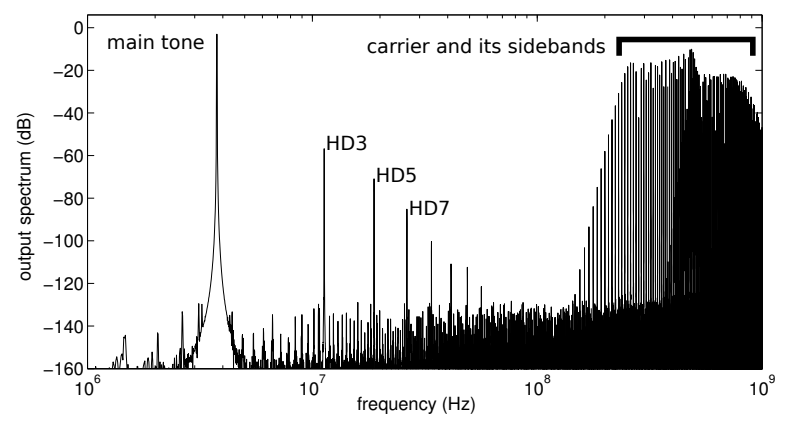

Fig. 1. A typical spectrum for the output of an ASDM.

On the other hand, [8] and [9] do focus on the same class of ASDMs as our manuscript. However, [8] concerns a simulation based aproach instead of an analytical one. The only work that presents analytical expressions to predict the 3rd order harmonic distortion of an ASDM is by Roza [9]. This work gives a good insight to the understanding and analysis of an ASDM, but the final equation for the distortion does not have a good match with simulation results. Therefore we will present a more accurate analysis underneath to find an expression for the distortion and carrier frequency of an ASDM, then we will compare the proposed theory with simulation results and with Roza's results [9] as well.

Fig. 1 shows a typical spectrum for the output of an ASDM with a single tone input signal. As Fig. 1 shows, the output of this modulator consists of baseband distortion components (the harmonics of the input tone: HD3, HD5, ...) and higher frequency spurs corresponding to the carrier frequency and its sidebands [9]. As it will be proven later, and as Fig. 1 confirms, ASDM has an odd nature, therefore, its output only contains odd order harmonics of its input signal. The proposed theory accurately estimates these harmonics with explicit expressions for the 3rd and the 5th harmonic.

If the carrier frequency is high enough, depending on the application of the ASDM (i.e. ADC or PA), the high frequency spurs can be filtered later in the digital or analog domain. Nevertheless, as we will see later, the carrier frequency of an ASDM is important in determining the distortion of the modulator. Moreover, it puts a limit on the bandwidth and power consumption. Therefore, estimation of the carrier frequency is also included in this work.

\section{THEORY}

In this section we will elaborate the proposed theory. First, an accurate theory is presented for a general input signal. Second, the theory is simplified, and third, it is applied to a 


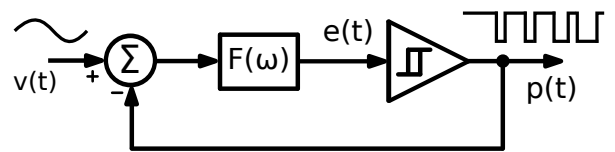

Fig. 2. A general representation of an ASDM.

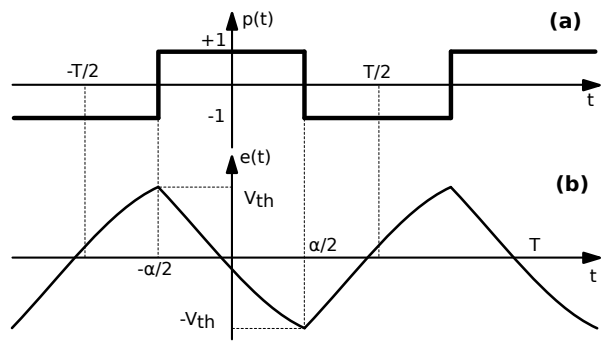

Fig. 3. Typical waveforms for the ASDM in Fig. 2.

special case where the input signal of the ASDM is sinusoidal. The harmonic distortion components and the carrier frequency for that case will then be derived.

\section{A. General Theory}

Fig. 2 shows the general diagram of an ASDM. Here, $v(t)$ is an arbitrary input signal and is assumed to be limited to a full scale of \pm 1 . $e(t)$ is the filtered error signal at the input of the schmitt-trigger. $p(t)$ is the output square wave with a pulse width of $\alpha$ and instantaneous period $T$, which toggles between +1 and -1 . As it will be shown later in this section, in this type of modulation, not only the duty cycle of the output square wave, $\frac{\alpha}{T}$, but also its carrier frequency are dependent on the input signal. The carrier frequency of an ASDM for a zero input is referred to as $\omega_{c}$ and $\omega_{1}=\frac{2 \pi}{T}$ is the (instantaneous) carrier frequency which is a function of the input signal, $v$. According to Fig. 2 we can write the signal flow at e(t):

$$
e(t)=(v(t)-p(t)) \otimes f(t)
$$

where $f(t)$ is the time domain impulse response of the filter, $F(\omega)$, and $\otimes$ is the convolution.

Assuming that the input bandwidth is much lower than $\omega_{1}$, then the input signal, $v$, can be considered constant during a few periods of $p(t)$ and $p(t)$, which is a square wave, can be described by its Fourier series:

$$
p(t)=\left(\frac{2 \alpha}{T}-1\right)+4 \times \operatorname{Re}\left\{\sum_{n=1}^{\infty} \frac{\sin \left(n \omega_{1} \frac{\alpha}{2}\right)}{n \pi} e^{j n \omega_{1} t}\right\}
$$

The $\left(\frac{2 \alpha}{T}-1\right)$ term in $p(t)$, which will be referred to as $u$ hereafter, is the baseband component of $p(t)$ which includes the desired signal and its harmonic distortion components, so we can say:

$$
u=\left(\frac{2 \alpha}{T}-1\right) \quad \text { or, } \quad \omega_{1} \alpha=\pi(u+1)
$$

Fig. 3 shows typical waveforms of an ASDM. As the figure shows, the schmitt-trigger toggles when $e(t)$ reaches its positive or negative thresholds, $V_{t h}$ or $-V_{t h}$. So if we use $t=\frac{-\alpha}{2}$ and substitute (2) in (1), we obtain:

$$
\begin{aligned}
& V_{t h}=(v-u) \otimes f-4 \sum_{n=1}^{\infty}\left(\frac{\sin \left(n \omega_{1} \frac{\alpha}{2}\right)}{n \pi}\right) \times \\
& {\left[\operatorname{Re}\left\{F\left(n \omega_{1}\right)\right\} \cos \left(n \omega_{1} \frac{\alpha}{2}\right)+\operatorname{Im}\left\{F\left(n \omega_{1}\right)\right\} \sin \left(n \omega_{1} \frac{\alpha}{2}\right)\right]}
\end{aligned}
$$

and for $t=\frac{\alpha}{2}$ we will have:

$$
\begin{aligned}
& -V_{t h}=(v-u) \otimes f-4 \sum_{n=1}^{\infty}\left(\frac{\sin \left(n \omega_{1} \frac{\alpha}{2}\right)}{n \pi}\right) \times \\
& {\left[\operatorname{Re}\left\{F\left(n \omega_{1}\right)\right\} \cos \left(n \omega_{1} \frac{\alpha}{2}\right)-\operatorname{Im}\left\{F\left(n \omega_{1}\right)\right\} \sin \left(n \omega_{1} \frac{\alpha}{2}\right)\right]}
\end{aligned}
$$

This result is only valid if we assume that $v$ is changing much slower than $p(t)$, that is $v\left(t=\frac{-\alpha}{2}\right) \approx v\left(t=\frac{\alpha}{2}\right)$. Subtracting (4) and (5) will result in:

$$
\sum_{n=1}^{\infty} \frac{1}{n} \sin ^{2}\left(n \omega_{1} \frac{\alpha}{2}\right) \operatorname{Im}\left\{F\left(n \omega_{1}\right)\right\}=-\frac{\pi}{4} V_{t h}
$$

and adding them will give:

$$
(v-u) \otimes f=\frac{2}{\pi} \sum_{n=1}^{\infty} \frac{\operatorname{Re}\left\{F\left(n \omega_{1}\right)\right\}}{n} \sin \left(n \omega_{1} \alpha\right)
$$

Solving (6) will give an expression for the oscillation frequency, $\omega_{1}$, as a function of $u$. Afterwards, solving (7) will then give the baseband component of the output signal, $u$, as a function of the input signal, $v$.

In order to solve the series in (6) and (7), we should have an understanding about the loop filter. In most of the conventional filters that are generally used in ASDMs (like the ones used in the next section as examples or in [9]) for $\omega \geqslant \omega_{1}$ we have:

$$
\operatorname{Re}\{F(\omega)\} \approx \frac{1}{\left(\tau_{r} \cdot \omega\right)^{2}}
$$

and,

$$
\operatorname{Im}\{F(\omega)\} \approx \frac{1}{\tau_{i} \cdot \omega}
$$

where $\tau_{r}$ and $\tau_{i}$ are filter dependent constants. We will see that these assumptions are valid if the poles and zeros of the filter are much lower than $\omega_{1}$. In this manner, the filter will have about $90^{\circ}$ phase shift around the carrier frequency. The additional $90^{\circ}$ phase shift required in the loop for the oscillation to take place, will be provided by the hysteresis of the schmitt-trigger.

Based on the assumption in (9), the series in (6) can be simplified using [12, p. 92, Eq. 4], which results in:

$$
\omega_{1}=\omega_{c}\left(1-u^{2}\right) \quad \text { with }, \quad \omega_{c}=\frac{\pi}{2 \tau_{i} \cdot V_{t h}}
$$

In order to solve (7), we will first simplify it by substituting $\omega_{1} \alpha=\pi(u+1)$, according to (3). Then, considering the assumption of (8), the series in (7) can be evaluated using [12, p. 87 , Eq. 4]:

$$
\sum_{n=1}^{\infty} \frac{\operatorname{Re}\left\{F\left(n \omega_{1}\right)\right\}}{n} \sin \left(n \omega_{1} \alpha\right)=\frac{-\pi^{3}}{12} \frac{\left(u-u^{3}\right)}{\left(\tau_{r} \cdot \omega_{1}\right)^{2}}
$$


Since $\omega_{1}$ is also a function of $u$, in the right side of (11) we can substitute $\omega_{1}=\omega_{c}\left(1-u^{2}\right)$ from (10). Now by inserting (11) in (7) we will have:

$$
(v-u) \otimes f=\frac{2}{\pi} \frac{-\pi^{3}}{12} \frac{\left(u-u^{3}\right)}{\left(\tau_{r} \cdot \omega_{c}\left(1-u^{2}\right)\right)^{2}}
$$

and taking (8) into account, for $\omega_{c}$, will give:

$$
(u-v) \otimes f=\frac{\pi^{2}}{6} \operatorname{Re}\left\{F\left(\omega_{c}\right)\right\} \frac{u}{1-u^{2}}
$$

Eq. (13) gives a very accurate relationship between the input signal, $v$, and the baseband output signal, $u$. Hence, in principle, all non-linearity effects of the modulator could be derived from this equation using numerical methods. After obtaining $u$ from this equation, the instantaneous carrier frequency can also be accurately calculated using (10).

Eq. (13) also allows to make an important observation: if the real part of the loop filter transfer is zero, the modulation process does not generate in-band harmonic distortions. This occurs when the loop filter is an integrator. This was also already observed in [9] and hence in terms of modulation distortion, an integrating loop filter is the optimal choice.

\section{B. Approximate Theory}

Eq. (13) gives $u$ as an accurate yet implicit function of $v$. Usually, we prefer to have an explicit analytical expression so that we don't need numerical methods to solve it. For this, we use Perturbation theory: first we make a rough and 1st order estimation of the output signal, $u$, then we use that estimation to simplify the equation, solve it again, and find a more accurate answer for $u$.

By observing that the term on the right hand of (13) is much smaller than the ones on the left hand, we obtain in a first approximation that $u \approx v$. Now, we can use this approximation to estimate the contribution of the right hand of (13) and we obtain the following improved approximation:

$$
(u-v) \otimes f \approx \frac{\pi^{2}}{6} \operatorname{Re}\left\{F\left(\omega_{c}\right)\right\} \frac{v}{1-v^{2}}
$$

Eq. (10) also gives a very accurate expression for $\omega_{1}$. But, since we prefer to have $\omega_{1}$ as a function of the input signal $v$, rather than the output signal $u$, we can again use the approximation $u \approx v$, which gives the following simplified expression:

$$
\omega_{1} \approx \frac{\pi\left(1-v^{2}\right)}{2 \tau_{i} \cdot V_{t h}}
$$

\section{Sinusoidal input signal}

Now we will apply the approximate theory to the case where the input signal is a single tone, $v=A \sin (\mu t)$, to obtain the harmonic distortion components of the modulator. By assuming $v=A \sin (\mu t)$, the right side of (14) can be expanded using its Fourier series. Then we will have:

$$
(u-v) \otimes f=\frac{\pi^{2}}{6} \operatorname{Re}\left\{F\left(\omega_{c}\right)\right\} \sum_{n=1}^{\infty} \mathrm{K}_{n}(A) \sin (n \mu t)
$$

where $\mathbf{K}_{n}(A)$ comes from this Fourier integral:

$$
\mathrm{K}_{n}(A)=\frac{\mu}{\pi} \int_{\frac{-\pi}{\mu}}^{\frac{\pi}{\mu}} \frac{A \sin (\mu t) \cdot \sin (n \mu t)}{1-(A \sin (\mu t))^{2}} d t
$$

It can be shown that the integral in $\mathrm{K}_{n}(A)$ is zero for even values of $n$. Therefore, according to (16), the Fourier expansion of $u$ should also include only the odd harmonics of the input frequency, $\mu$. So the Fourier expansion of $u$ will be:

$$
u=\sum_{n} a_{n} \cdot \sin \left(n \mu t-\theta_{n}\right), \quad n=1,3,5, \ldots
$$

By substituting (18) in (16), we obtain the phase $\theta_{n}$ and the amplitude $a_{n}$ for each harmonic by matching the corresponding $\sin (n \mu t)$ terms from both sides of the equation. For the main tone, $n=1$, since the terms on the right hand of (16) are much smaller than the main tones on the left, we can simply deduce that $a_{1} \approx A$. For the harmonics we obtain:

$$
\theta_{n}=\angle[F(n \mu)], \quad a_{n} \cdot|F(n \mu)|=\frac{\pi^{2}}{6} \operatorname{Re}\left\{F\left(\omega_{c}\right)\right\} \mathrm{K}_{n}(A)
$$

and from that we can finally obtain the ratio of the magnitude of the nth harmonic over the main tone:

$$
d_{n}=\frac{a_{n}}{a_{1}} \approx \frac{a_{n}}{A}=\frac{\pi^{2}}{6} \frac{\mathrm{K}_{n}(A)}{A} \frac{\operatorname{Re}\left\{F\left(\omega_{c}\right)\right\}}{|F(n \mu)|}
$$

Eq. (17) for the $\mathrm{K}_{n}$ factors can also be calculated. E.g. for the 3rd and 5th harmonic this leads to (for $A \leq 1$ ):

$$
\begin{gathered}
\mathrm{K}_{3}(A)=\frac{2 A^{2}-8}{-A^{3}}+\frac{6 A^{2}-8}{A^{3} \sqrt{1-A^{2}}} \\
\mathrm{~K}_{5}(A)=\frac{2 A^{4}-24 A^{2}+32}{-A^{5}}+\frac{10 A^{4}-40 A^{2}+32}{A^{5} \sqrt{1-A^{2}}}
\end{gathered}
$$

By substituting (21) in (20), we obtain the 3 rd order harmonic distortion of an ASDM:

$$
d_{3}=\frac{\pi^{2}}{6}\left(\frac{2 A^{2}-8}{-A^{4}}+\frac{6 A^{2}-8}{A^{4} \sqrt{1-A^{2}}}\right) \frac{\operatorname{Re}\left\{F\left(\omega_{c}\right)\right\}}{|F(3 \mu)|}
$$

The average carrier frequency of the modulator, $\overline{\omega_{1}}$, can be accurately calculated by averaging (10). But it is easier to use the estimation in (15). For a sinusoidal input, $v=A \sin (\mu t)$, the average carrier frequency will be:

$$
\overline{\omega_{1}}=\frac{\pi\left(1-0.5 A^{2}\right)}{2 \tau_{i} \cdot V_{t h}}
$$

Except for the accurate value of $\omega_{1}$ in (10), the other approximations for $\omega_{c}, \omega_{1}$, and $\bar{\omega}_{1}$ in (10), (15), and (24) have been previously reported [2], [6]-[9].

\section{Simulation Results}

To verify the proposed theory, the ASDM in Fig. 2 was simulated in Simulink for two cases, once with a 1st order passive loop filter and then with a 3rd order active one. The simulation results for the 3rd order harmonic distortion were also compared with a previous theory by Roza [9] which gave:

$$
d_{3}[R o z a]=\frac{\pi^{2} A^{2}}{6} \frac{\operatorname{Re}\left\{F\left(\overline{\omega_{1}}\right)\right\}}{F(\mu)}
$$


Here, $d_{3}[$ Roza $]$ comes from the series expansion of the output, $u \approx v+d_{3} v^{3}$. This result is frequently misinterpreted as if $d_{3}[R o z a]$ corresponds to the 3rd harmonic distortion component, this is incorrect because the cube of a sine wave equals:

$$
\sin ^{3} x=\frac{1}{4}(3 \sin x-\sin 3 x)
$$

This way, the correct 3rd harmonic distortion predicted by [9] equals $d_{3}[R o z a] / 4$, corresponding to a difference of $12 \mathrm{~dB}$.

\section{A. First order passive loop filter}

As a first example, an ASDM with a 1st order passive loop filter, $F(s)=\frac{1}{1+s \tau}$, was evaluated. By mapping this to (8) and (9) we obtain $\left|\tau_{i}\right|=\left|\tau_{r}\right|=\tau$. We have chosen $\tau=5$ ns. To have a usable ASDM the threshold voltage $V_{t h}$ should be sufficiently small, therefore it was set to $V_{t h}=0.1$ which corresponds to a (zero-input) self oscillation frequency, $f_{c}=$ $\frac{\omega_{c}}{2 \pi}=500 M H z$, according to (10). Different simulation setups were employed to examine different aspects of the theory.

In the first set of simulations, a DC signal has been applied to the input of the modulator. Both the DC level of the output and its carrier frequency have been evaluated. The output DC level has been compared both with the accurate theory of (13) and the approximate theory of (14). Take in mind that for DC signals we have $F(0)=1$, and $\operatorname{Re}\left\{F\left(\omega_{c}\right)\right\}$ can be obtained by combining (8) and (10), so (13) will collapse to:

$$
v=u-\frac{2 V_{t h}^{2}}{3}\left(\frac{u}{1-u^{2}}\right)
$$

and by doing the same to (14) we will have:

$$
u \approx v+\frac{2 V_{t h}^{2}}{3}\left(\frac{v}{1-v^{2}}\right)
$$

As Fig. 4 (a) shows, there is a perfect match between the accurate theory in (27) and the simulation results. The curve corresponding to the approximate theory, (28), also has a good match and only a little deviation for higher input DC values. The same conclusion applies to Fig. 4 (b) where the carrier frequency has been compared with the accurate theory of (10) and the approximate theory of (15).

In the next set of simulations, a sinusoidal input with an amplitude of $A=0.7$ is applied to the aforementioned modulator and the 3rd harmonic of the output is evaluated for a range of input frequencies. According to (24), with this input amplitude, an average carrier frequency of $\overline{\omega_{1}} \approx 377 \mathrm{MHz}$ is expected. Fig. 5 shows the corresponding simulation results. The plot also shows the proposed theory [Eq. (23)]. For comparison with [9], two curves have been added. The first corresponds to the incorrect interpretation of Roza's theory [i.e. use (25) for the 3rd harmonic distortion] and the other adds $-12 d B$ to it to compensate for the $\frac{-1}{4}$ factor according to (26) and hence corresponds to a correct interpretation of Roza's theory. As this set of simulations confirms, the proposed theory predicts the distortion of an ASDM much more accurately than [9].

Fig. 6 illustrates the dependency of the (average) carrier frequency and the 3rd order harmonic distortion on the input amplitude, $A$, according to simulation and according to (24) and (23) for the case of an arbitrary input frequency of $\mu=$

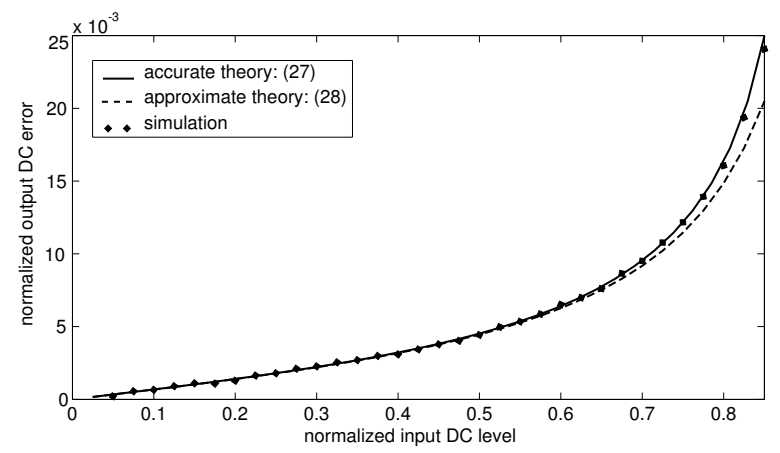

(a)

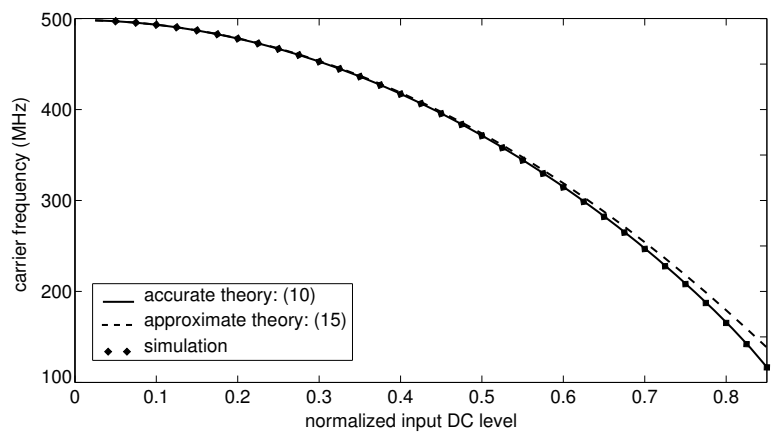

(b)

Fig. 4. Comparison between simulation results and theory for a 1st order ASDM with a DC input: (a) the difference between the DC level of output and input signals, and (b) the carrier frequency.

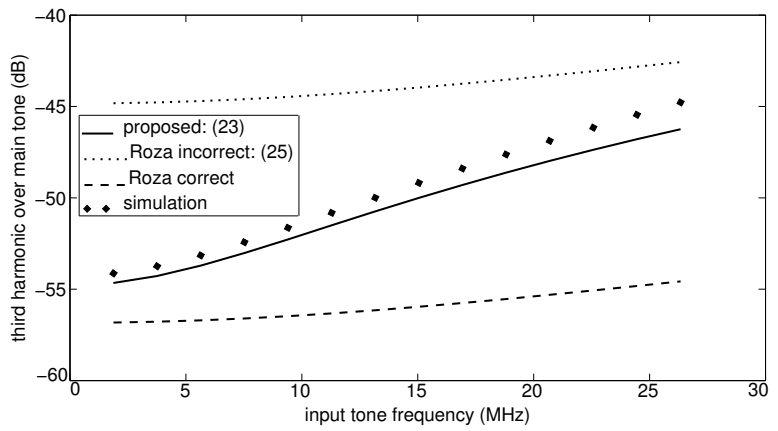

Fig. 5. Comparison between simulation results and theory for an ASDM with a 1 st order passive filter.

$12.5 \mathrm{MHz}$ over a range of input amplitudes. Agian the plot confirms a good match between the simulation and the theory.

\section{B. Active loop filters}

Many widely used active filters satisfy the conditions in (8) and (9), including the following family: $F(s)=\frac{\prod_{j=1}^{n-1}\left(1+s \tau_{j}\right)}{\left(s \tau_{0}\right)^{n}}$. In the second example, we will investigate the 3rd order case of $n=3$, where we used the following ASDM parameters $\tau_{0}=\tau_{1}=\tau_{2}=5 n s$ and $V_{t h}=0.1$. By mapping the given transfer function to (8) and (9) we will have $\left|\tau_{i}\right|=5 n s$ and $\left|\tau_{r}\right|=\frac{5 n s}{\sqrt{2}}$. According to (10), these values correspond to a self oscillation frequency of $f_{c}=500 \mathrm{MHz}$.

In the first set of simulations, the 3rd order harmonic distortion of the modulator has been compared with the proposed theory in (23) and [9]. The input has been given an arbitrary amplitude of $A=0.7$ and the result has been drawn 


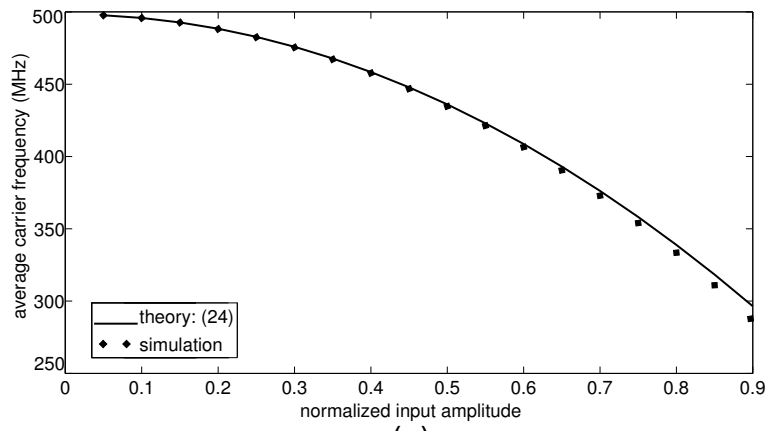

(a)

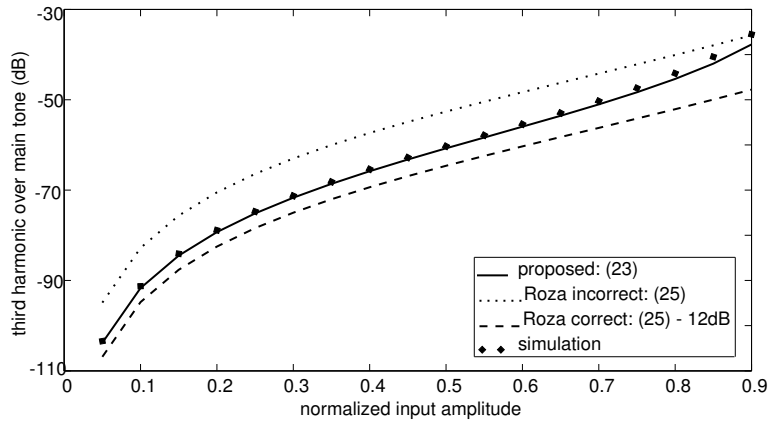

(b)

Fig. 6. Average carrier frequency, $\overline{f_{c}}$, and the 3 rd order distortion, $d_{3}$, of a 1 st order ASDM as a function of its input amplitude $A$.

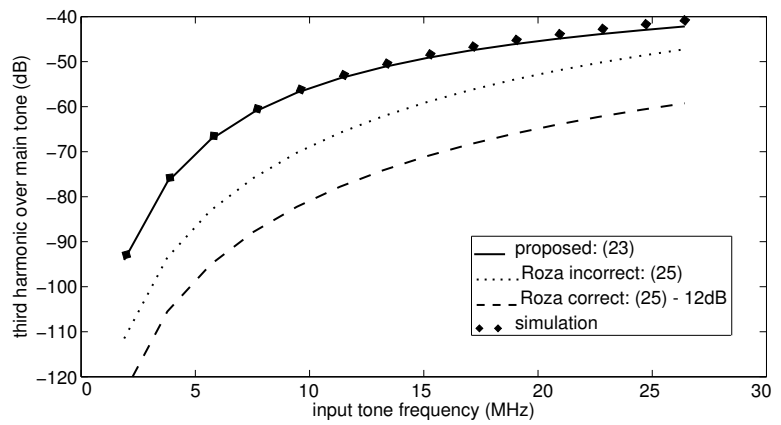

Fig. 7. Comparison between simulation results and theory for the 3rd order harmonic distortion of a 3rd order ASDM over its input frequency.

over a range of input frequencies. As in previous example, two curves have been drawn corresponding to the correct and incorrect interpretations of Roza's theory. As Fig. 7 shows, the proposed equation matches the simulation results almost perfectly and [9] (the curve tagged as "Roza correct") has an average error of more than $20 \mathrm{~dB}$.

In the last set of simulations, for an arbitrary input frequency of $\mu=12.5 \mathrm{MHz}$, the average carrier frequency and the 3rd order harmonic distortion were evaluated for varying input amplitudes and the results were compared with the proposed theory according to (24) and (23). Fig. 8 shows the corresponding results. Once again there is a good match between the simulation and theory.

\section{CONCLUSION}

We have derived Eq. (23) to predict the 3rd harmonic distortion of an ASDM. The analytical results matched very well with simulations and showed considerable improvement

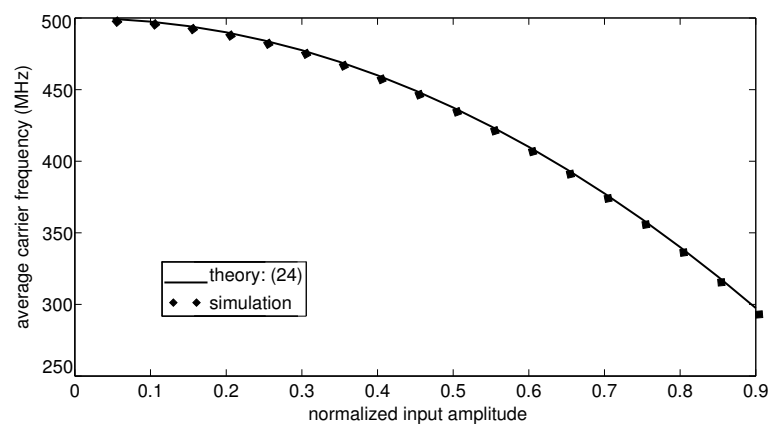

(a)

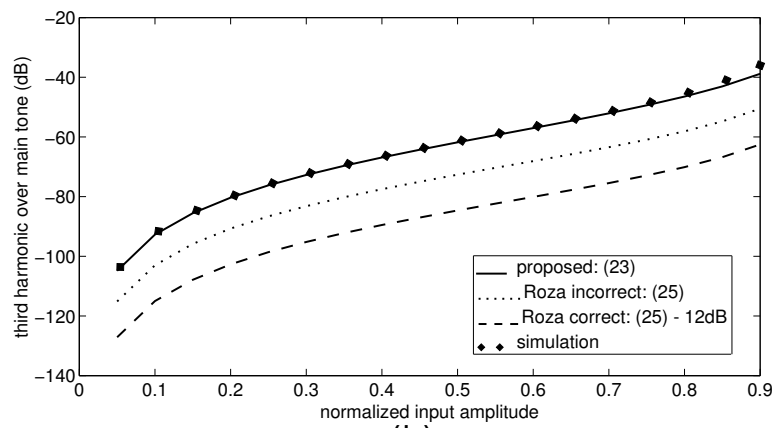

(b)

Fig. 8. Average carrier frequency, $\overline{f_{c}}$, and 3rd order distortion, $d_{3}$, of a 3rd order ASDM vs. the input amplitude, $A$.

over the prior art. The results of this work can be used in design an optimization of schmitt trigger based ASDMs.

\section{REFERENCES}

[1] L. Hernandez, S. Paton, and E. Prefasi, "VCO-based sigma delta modulator with PWM precoding," Electron. Lett., vol. 47, no. 10, pp. 588-589, May 12, 2011.

[2] J. Daniels, W. Dehaene, M. Steyaert, and A. Wiesbauer, "A/D conversion using an asynchronous delta-sigma modulator and a time-to-digital converter," in IEEE Int. Symp. Circuits and Syst. (ISCAS), May 2008, pp. $1648-1651$

[3] A. Babaie Fishani and P. Rombouts, "Continuous time Delta Sigma modulation with PWM pre-coding and binary $\mathrm{g}(\mathrm{m})$ blocks," Electron. Lett., vol. 48, no. 19, pp. 1187-1188, Sep 132012.

[4] B. De Vuyst and P. Rombouts, "A 5-MHz 11-Bit Self-Oscillating Sigma Delta Modulator With a Delay-Based Phase Shifter in $0.025 \mathrm{~mm}^{2}$," IEEE J. Solid-State Circuits, vol. 46, no. 8, pp. 1919-1927, Aug. 2011.

[5] M. Berkhout, "Class-D audio amplifiers in mobile applications," in IEEE Int. Symp. Circuits and Syst. (ISCAS), pp. 1169 -1172, May 2009.

[6] G. Roberts and M. Ali-Bakhshian, "A brief introduction to time-todigital and digital-to-time converters," IEEE Trans. Circuits Syst.-II, vol. 57 , no. 3, pp. $153-157$, Mar. 2010.

[7] T. Matic, T. Svedek, and M. Herceg, "A method for the schmitttrigger propagation-delay compensation in asynchronous sigma delta modulator," IEEE Trans. Circuits Syst.-II, vol. 59, no. 7, pp. $404-408$, Jul. 2012.

[8] S. Ouzounov, E. Roza, J. Hegt, G. van der Weide, and A. van Roermund, "Analysis and design of high-performance asynchronous sigma-delta modulators with a binary quantizer," IEEE J. Solid-State Circuits, vol. 41, no. 3, pp. 588-596, Mar. 2006.

[9] E. Roza, "Analog-to-digital conversion via duty-cycle modulation," IEEE Trans. Circuits Syst.-II, vol. 44, no. 11, pp. 907-914, Nov 1997.

[10] T. Piessens and M. Steyaert, "Behavioral analysis of self-oscillating class D line drivers," IEEE Trans. Circuits Syst.-I, vol. 52, no. 4, pp. 706 714, Apr. 2005.

[11] F. Colodro and A. Torralba, "Spectral Analysis of Pulsewidth-Modulated Sampled Signals," IEEE Trans. Circuits Syst.-II, vol. 57, no. 8, pp. 622626, Aug. 2010.

[12] V. Mangulis, Handbook of series for scientists and engineers, ser. Pure and Applied Physics. Academic Press, 1965. 